\title{
The Costs of Industry-Sponsored Medical Device Clinical Trials in Alberta
}

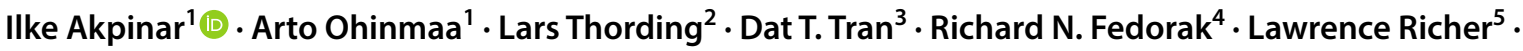 \\ Philip Jacobs ${ }^{4}$
}

Published online: 29 April 2019

(c) The Author(s) 2019

\begin{abstract}
Objective Our objective was to describe the costs of industry-sponsored clinical trials for medical devices in Northern Alberta, Canada.

Methods We used centralized data to identify all industry-sponsored medical device clinical trials initiated in Northern Alberta from 2012 to 2016. For each arm of each trial, we calculated the price of devices provided by the sponsor and the cost of clinical and administrative services that were incurred to clinically operationalize the treatment.

Results Our sample consisted of 18 device trials initiated between January 2012 and January 2016. The overall cost (Canadian dollars [ $\$$ Can], year 2018 values) per enrolee was $\$$ Can 18,243 for the experimental arm and $\$$ Can13,827 for the control arm. Devices were the highest cost component, at $\$$ Can13,446 per enrollee in the experimental arm. Clinical costs in the control arms were higher on average ( $\$$ Can7202 vs. 2504) than those in the experimental arms.

Conclusion Data from industry-sponsored clinical trials can provide important information on the full costs of device-related interventions. As device costs rise, and as policy makers require more evidence on device-related treatments, the cost of medical device-driven interventions should be documented along with their effectiveness.
\end{abstract}

\section{Key Points for Decision Makers}

The industry-sponsored medical device trials that were conducted during the study period were mainly singlearm trials and differed considerably in time (longer) and cost (lower) from drug clinical trials.

Per person, medical device trials averaged about \$Can13,000 for devices and \$Can18,000 overall in the experimental arm (year 2018 values).

Device trials can provide a key to understanding the full cost of treatments in which devices are a major component.

\section{Introduction}

Clinical trials (CTs) are important components in the licensing of medical devices [1, 2]. In the USA, the FDA requires manufacturers to conduct clinical studies to obtain a premarket approval for class III devices. In Canada, evidence of effectiveness from CTs is required for class IV medical devices [1]. Health Canada is responsible for issuing approvals to allow trials with a medical device to be conducted in Canada [2]. The FDA class III and Health Canada class IV devices are highly invasive in nature. The design of a medical device CT differs from that of a drug CT: randomization is not common for medical device

Richard N. Fedorak: Deceased 8 November 2018.

Ilke Akpinar

ilke@ualberta.ca

1 School of Public Health, University of Alberta, 3-267 Edmonton Clinic Health Academy, 11405-87 Ave, Edmonton, AB T6G 1C9, Canada

2 Innovative Health, Scottsdale, AZ, USA
3 Faculty of Pharmacy and Pharmaceutical Sciences, University of Alberta, Edmonton, AB, Canada

4 Department of Medicine, University of Alberta, Edmonton, AB, Canada

5 Department of Pediatrics, University of Alberta, Edmonton, AB, Canada 
CTs and it is difficult to ensure blinding for individuals or investigators in a device trial. Medical device CTs are usually conducted with smaller patient populations [3] and have mostly been designed to generate endpoints to meet the approval requirements of the regulatory agencies [4].

Although studies exist that document the economics of clinical research, these are for pharmaceuticals or medical and surgical procedures [5-8]. The research, development, and approval process is much more developed for drugs than for medical devices, although recent news reports by an international coalition of investigative journalists called for increased clinical research on specific devices [9]. Prior to this call for evidence, the importance of clinical research on devices had already been growing. According to the US National Library of Medicine database, ClinicalTrials.gov, the number of medical device trials started in the USA increased from 51 in 2005 to 139 in 2015 [10]. Although the US FDA and Health Canada do not require economic data in their approval submissions, the rapid growth of expenditure on a variety of medical devices [11], and the growing requirements for industryprovided evidence, calls for a greater degree of economic considerations in CTs.

In 2017, the revenues of the top ten medical device companies ranged from $\$ \mathrm{Can} 28.8$ billion to 10.1 billion globally [12], and these companies spent about $10 \%$ of their revenues on research [13]. Even though we know the amount that the industry spends on total research and development, almost no information is available on expenditure and costs of clinical (as opposed to basic) research for medical devices. Clinical research is the medium through which devices are formally tested and compared with alternative treatments. Economic aspects of clinical research provide important information to policy makers on the full cost of alternative interventions, not just on the device costs themselves. As CTs also impose a burden on developers, it is important to have an estimate of the magnitude of this.

There is growing interest among policy makers to increase the scrutiny of the performance of new technologies, including medical devices. CTs will play a major role in this movement. The purpose of this study is to describe the costs of industry-sponsored CTs for medical devices in Northern Alberta, including the types of trials and their costs of devices and associated clinical services.

\section{Methods}

\subsection{Environment}

The healthcare system in Alberta is a single, province-wide, fully integrated institutional service provided by Alberta
Health Services (AHS) [14]. Alberta health authorities and hospitals provide approximately $70 \%$ of medical device funding [1]. Some devices are purchased or funded through a specialty program led by the provincial health ministry. The Alberta Aids to Daily Living Program also provides financial assistance for the purchase of medical equipment for people on low incomes with long-term physical disabilities or chronic or terminal illnesses [1].

The study sample data were obtained from the Northern Alberta Clinical Trials and Research Center (NACTRC) - a joint venture between the University of Alberta and AHS [15]. NACTRC is responsible for negotiating CT agreements and research approvals in Northern Alberta. Data describing the $\mathrm{CT}$, including clinical protocols and budgets, are maintained in a central database. Clinical services budgets and overheads that go into the contracts are set by AHSFinance and reflect actual costs. Research teams directly submit their invoices to the industrial trial sponsors. Payments from the sponsors are received and deposited in AHS accounts. Investigators and their teams manage expenses and payment tracking and make reimbursement (e.g., salary) submissions to AHS-Finance for team activities (research staff are employees of AHS) [16].

To describe the costs of industry-sponsored medical device CTs, we included the following variables in our analysis: the market value of the medical devices used in the trials, revenues billed to the industry for clinical and other services, and administrative expenses of CT sites for each trial.

\subsection{Trial Sample}

Our target sample included all industry-sponsored medical device CTs that were processed through NACTRC and initiated between January 2012 and January 2016. We chose 30 January 2018 as the end date for our analysis, and the start date was chosen to allow sufficient time for the trials to end. We obtained the trial budgets from NACTRC and the number of patients enrolled in each trial from the University of Alberta Research Ethics Board.

\subsection{Medical Device Pricing}

In most instances, neither manufacturer list prices nor actual prices were publicly available. Actual prices can be lower than list prices because providers negotiate discounts with the suppliers. The absence of actual prices poses difficulties for people conducting economic evaluations of CTs. We used the CT protocols from the website ClinicalTrials.gov [10] to identify the devices used in the study then developed a hierarchical method to identify the price of each device, 
similar to the method used by Akpinar et al. [17]. Our pricing algorithm is shown in Fig. 1. First, we examined the CT budgets to see whether a device price was provided; if not, we requested it from the manufacturer. If a price was not found in the budget document and the manufacturer did not provide the list price, we used the US (original equipment) price of the device based on US hospital pricing information collected by Innovative Health, a medical devicereprocessing company in Scottsdale, AZ, USA. These prices were actual, not listed, prices and were only available for selected cardiac devices. If a price was not found in Innovative Health's database, we obtained data from the published literature (using cost-effectiveness studies and health technology assessment reports). All prices were converted to Canadian dollars (\$Can), year 2018 values, using purchasing power parity measures obtained from Organisation for Economic Co-operation and Development data [18]. The device cost for placebo treatments was set to nil. If a device was in the premarket phase, we dropped it from the sample as no price was available.

\subsection{Estimating Industry Billings for Clinical Services}

We used trial budgets to measure billings for each trial. We measured service costs according to trial protocols and categorized billed services into three major groups: (1) management billings (including start-up costs, storage of study device, document preparation and archival costs), (2) research ethics board fees, and (3) patient service billings (laboratory tests and imaging, procedures, consultations, treatment of adverse events). Budgeted rates included direct service costs plus estimated overheads. Control arms

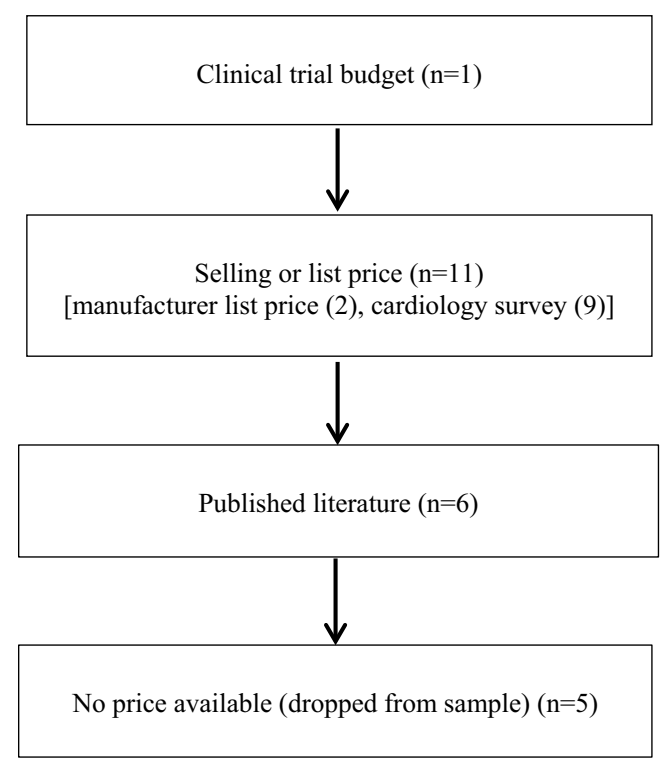

Fig. 1 Algorithm for pricing trial device considered all relevant comparators, including trial devices, other devices, and current standard care for patients. If the current standard care was a procedure, such as a surgery, its cost was included in the patient service billings.

We did not include inflation adjustments for device prices because of the way the medical device market works. It is expected that the device price would decrease or stay stable considering rapid technological development and the short lifetimes of the devices [19]. For the trial budgets, we applied the inflation rates using the all-item Consumer Price Index for Alberta [20].

Total economic values were calculated for trials in four major categories: administrative billings (including start-up costs, storage of study device, document preparation and archival costs), ethics review fees, patient service billings (e.g., laboratory tests and imaging, procedures, consultations, treatment of adverse events), and device costs. Service billings were calculated by arm: experimental arm only for single-arm studies; experimental and control arms for others.

We also calculated the length of each CT in months from its start date to its end date or to our study end date of January 2018, whichever came first, and reported the CT lengths in months ( \pm standard deviation). All analyses were performed using Microsoft Excel version 2016 (Microsoft Corp., Redmond, WA, USA).

\section{Results}

We obtained a list of 54 medical device CTs from NACTRC; $31(57 \%)$ of these were industry sponsored. We excluded a further three trials that did not use medical devices but were specifically designed to develop a clinical tool or evaluate a disease-specific patient-reported outcome instrument or a rehabilitation assessment program. In total, 28 industrysponsored medical device trials were initiated between January 2012 and January 2016.

Budget information was not available for 3 of $28 \mathrm{CTs}$, so we obtained complete data on 25 medical device trials. We excluded two medical device trials because of zero enrollment. We could not obtain market prices for five devices that were not yet on the market. Trials of 18 devices remained for analysis. Figure 2 shows the sample's disposition.

Of the 18 medical device trials remaining for analysis, the most common device group was cardiology, with ten (56\%) devices. Other specialties included pediatrics (two trials) and one each for gastroenterology, critical care, nephrology, radiology, emergency medicine, and dentistry. The ten cardiology devices varied widely in use and design. They included cryoablation catheters (two trials), implantable cardioverter defibrillators (two trials), a quadripolar left ventricular lead, a renal denervation system, a drug-eluting stent, a four-site lead/header system, a 
Fig. 2 Disposition of trials. NACTRC Northern Alberta Clinical Trials and Research Center

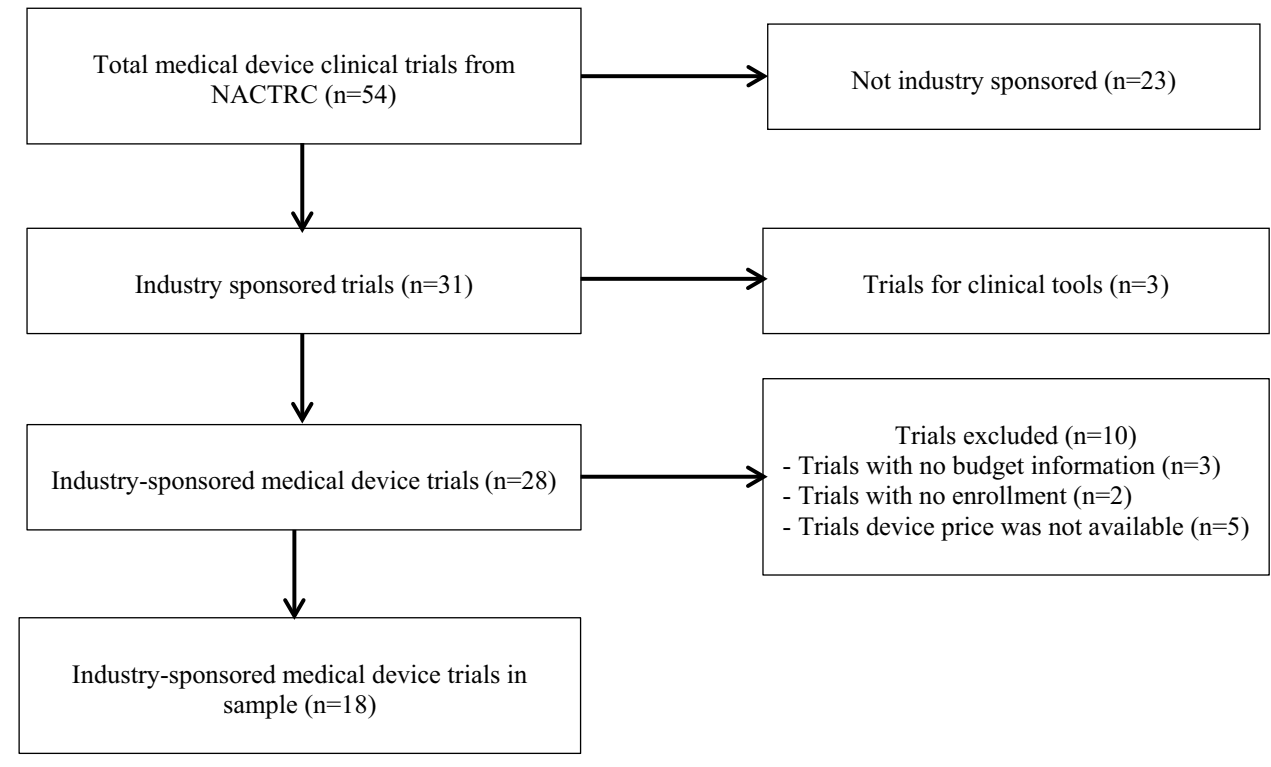

defibrillation lead, and a multipoint pacing cardiac resynchronization therapy defibrillator. The other eight devices came from various specialty areas, including pediatric cardiology (transcatheter pulmonary valve), pediatric endocrinology (automated drug-delivery device), dentistry (ultrasound-based device for orthodontic treatment), emergency medicine (noninvasive vagus nerve-stimulation device), interventional radiology (radiotherapy treatment device for hepatocellular carcinoma), nephrology (home hemodialysis machine), critical care medicine (cartridge for extracorporeal treatment), and gastroenterology (biliary stent system).

In medical device CTs, experimental arm patients always used the interventional device, whereas the control arm patients-could use similar devices from other companies, the interventional device, or no device, depending on the trial protocol. Of the devices, $16(89 \%)$ were classified as minimally invasive by the manufacturer. Nine $(50 \%)$ were implantable and $14(78 \%)$ were classified as "single use". All devices in our study were for treatment.

Six of the 18 included studies (33\%) were randomized controlled trials (RCTs), 11 (61\%) were single-arm studies, and one was a cohort study. In January 2018, seven of the CTs were closed and 11 were still active. The mean duration for all trials was 44.8 months $( \pm 18.2$, minimum 6 months, maximum 66 months) up to the end of our study in January 2018. The mean duration for the completed trials was 38 months $( \pm 20.7$, minimum 6 months, maximum 66 months). Table 1 presents the number of participants in the experimental and control arms.

One device price was obtained from the project budget, 11 from industry sources, and six from the literature [21-28]. We derived the device price information from different locations: ten from the USA, three from Canada, four
Table 1 Number of patients in the experimental and control arms

\begin{tabular}{|c|c|c|c|c|}
\hline \multirow[t]{2}{*}{ Department } & \multirow[t]{2}{*}{ Trials $(N)$} & \multirow{2}{*}{$\begin{array}{l}\text { Total } \\
\text { enroll- } \\
\text { ment }\end{array}$} & \multicolumn{2}{|c|}{ Patient numbers } \\
\hline & & & $\begin{array}{l}\text { Experi- } \\
\text { mental } \\
\text { arm }\end{array}$ & Control arm \\
\hline $\begin{array}{l}\text { Pediatric endocrinol- } \\
\text { ogy }\end{array}$ & 1 & 12 & 12 & \\
\hline Pediatric cardiology & 1 & 3 & 3 & \\
\hline Dentistry & 1 & 19 & 10 & 9 \\
\hline Emergency medicine & 1 & 4 & 4 & \\
\hline Nephrology & 1 & 5 & 5 & \\
\hline Radiology & 1 & 13 & 7 & 6 \\
\hline Critical care medicine & 1 & 13 & 7 & 6 \\
\hline Gastroenterology & 1 & 78 & 78 & \\
\hline Cardiology & 10 & 252 & 201 & 51 \\
\hline Total & 18 & 399 & 327 & 72 \\
\hline
\end{tabular}

a36 (50\%) control group patients (six critical care, nine dentistry, and 21 cardiology) used medical devices

from the UK, and one from Australia. Table 2 presents the trial costs. The overall mean cost per enrollee over all 18 trials was $\$$ Can 18,243 for experimental arms and $\$$ Can 13,827 for control arms. Devices were the highest cost component, at an average of $\$$ Can13,446 (74\% of total cost) per enrollee in the experimental arm. Clinical services formed the second-largest category in this arm. Clinical costs in the control arms were the major category, with $52 \%$ of the total cost per enrollee. Clinical costs were higher on average (\$Can7202 vs. 2504) in the control arm than in the experimental arms, primarily because the use of devices resulted in avoidance of more costly surgeries. For example, one of the trials in this analysis compared outcomes in patients with unprotected left main coronary artery disease treated with coronary 
Table 2 Average value per enrollee in each cost category of the medical device clinical trials

\begin{tabular}{lcc}
\hline Variable & $\begin{array}{c}\text { Number of enrollees } \\
\text { Average value per } \\
\text { enrollee (\$Can) }\end{array}$ \\
\hline Management billings & & 1901 \\
Ethics review fees & 327 & 392 \\
Clinical services (experimental arm) & 72 & 2504 \\
Clinical services (control arm) & & 7202 \\
Device costs (experimental arm) & 13,446 \\
Device costs (control arm) & & 4332 \\
Total clinical trial cost per enrollee (experimental arm) & & 18,243 \\
Total clinical trial cost per enrollee (control arm) & 399 & 13,827 \\
Overall for all enrollees & & \\
\hline
\end{tabular}

363 patients ( 327 from the experimental arm and 36 from control arm) used medical devices artery bypass graft or percutaneous coronary intervention using drug-eluting stents.

\section{Discussion}

We estimated the cost of the industry-sponsored medical device CTs that started in Northern Alberta between 2012 and 2016. Our data were based on device retail prices and trial budgets (for non-device costs). The average costs per enrollee were \$Can18,243, and \$Can13,827 in the experimental and control arms, respectively. Clinical costs were \$Can2504 and \$Can7202 for the experimental and control arms, respectively.

The devices in our sample varied widely in terms of specialty, cost, and trial design. This limited the degree to which we can generalize from our results. The device's market price is the price that the consumer or the insurer would actually pay for the device. In many cases, the retail price of the device was bundled in with the entire treatment cost, so the "wholesale" (provider) price was used. For each device, the transacting arrangements between clinical organization and device manufacturer vary considerably, so there can be a substantial variation in market prices. For ten devices, notably those in cardiology, we were able to obtain an average price paid (though they varied between hospitals) but needed to find proxies for market price in most other cases. In five trials, we could not get device prices at all, as the devices were not yet on the market.

In addition, new medical device prices decrease over time because of the rapid technological development and the short lifetimes of the devices [29]. The device price is usually quite high when it first comes to the market, so the total device costs of industry-sponsored device CTs could be substantially higher than when competition takes its toll on device prices. We also used prices for the USA, the UK, and Australia where we could not find Canadian prices. The medical device prices differ between these countries, and prices are higher in the USA than in some European countries [29].

Our sample of 31 (before exclusions) industry-sponsored trials was about $8 \%$ of the entire Canadian national sample of 394 industry-sponsored medical device trials in the same period. The sample and its characteristics were very different from those of drug trials. First, there were many more industry-sponsored drug trials: in Alberta, in the same time period, 628 drug trials were newly registered. Of these, 452 (71.8\%) were randomized, whereas one-third of our sample were randomized. The drug trials had more participants and were much more costly. In Alberta, a study using a similar study method in the same period reported a mean cost for CTs on a per patient basis of \$Can68,974 for noncancer drug CTs and \$Can221,492 for the experimental arms of cancer drug trials. These costs were significantly higher than those in our results. The mean duration time for the medical device CTs in our sample was longer than for the pharmaceutical CTs (44.8 vs. 25.2 months for noncancer trials and 38.7 months for cancer trials). In most cases, the costly part of the intervention of the device trials was usually the procedure day. On the other hand, pharmaceutical CT enrollees use the drugs throughout the study period; if the study drug is a chemical compound/biologic/biosimilar product, that usually increases costs.

In our analysis, we only considered the industry contribution to research within the healthcare system, but medical device companies incur other research-related expenses in their clinical studies that we did not include, such as inhouse administrative costs, internal monitoring costs, and the cost of preparing and submitting CT applications and amendments to the regulatory agencies, etc. [30]. These items are part of the contribution to the research and development process. If we were measuring the full cost of CTs, we would want to include them as they are part of the economic burden of having to conduct clinical research. In addition, some costs in our trials would not 
occur under standard care, including AHS management costs and ethics costs. If we wanted to measure care costs under normal circumstances, they would be excluded from the analysis. However, they only represent about $13 \%$ of the trial costs.

The time duration of the trials in our sample may not represent the full time that the device was in use or has impacted the system. In particular, costs for any related adverse events occurring after the trial closed would not be included.

\section{Conclusion}

Industry-sponsored CTs have been primarily used to gauge the effectiveness and safety of medical devices. CTs can also be used to estimate the costs of device-driven treatments, which is of value to healthcare managers, who must work within fixed budgets. It is also important for policy makers, who demand evidence to understand the burden placed on product developers. In this paper, we estimated the costs of medical device trials, including the device and clinical components. As device costs have increased, so too have the costs of device-driven treatments. CTs can be a valuable tool to help managers understand the economic aspects of using devices and to help policy makers understand the burden of providing evidence.

Data availability Data on trial characteristics are publicly available through the website clinicaltrials.gov. The budget documents used for the current study are not publicly available because of confidentiality restrictions.

Acknowledgements The authors acknowledge the contribution of the following people who helped us with obtaining data: Scott Klarenbach, University of Alberta; Don Juzwishin, Alberta Health Services; Rae Emogene, NACTRC; and Kim Kordov, Research Ethics, University of Alberta.

Author Contributions IA, PJ, RF, and LR conceptualized the study. The data collection and the initial analysis was undertaken by IA with further input from PJ, LT and AO. IA and PJ drafted the initial manuscript with all authors subsequently contributing to further drafts. IA, AO, LT, DT, LR and PJ approved the final manuscript.

\section{Compliance with Ethical Standards}

Funding This research did not receive any specific grant from funding agencies in the public, commercial, or not-for-profit sectors.

Conflicts of interest I Akpinar, A. Ohinmaa, D.T. Tran, R.N. Fedorak, L. Richer and P. Jacobs have no conflicts of interest that are directly relevant to the content of this article. Dr. Thording works for a com- pany that manufactures and sells reprocessed single-use devices. His role does not conflict with $\mathrm{CT}$ results.

Open Access This article is distributed under the terms of the Creative Commons Attribution-NonCommercial 4.0 International License (http://creativecommons.org/licenses/by-nc/4.0/), which permits any noncommercial use, distribution, and reproduction in any medium, provided you give appropriate credit to the original author(s) and the source, provide a link to the Creative Commons license, and indicate if changes were made.

\section{References}

1. Husereau D, Arshoff L, Bhimani S, N. A. Medical device and diagnostic pricing and reimbursement in Canada. Institute of Health Economics 2015. Report No.: 978-1-926929-63-7 Contract No.: 23 May 2018.

2. Government of Canada. Health Canada. Drug and medical device approval overview. https://www.canada.ca/en/services/health/ drug-health-products/drug-medical-device-highlights-2017/appro val-overview.html Accessed 20 Nov 2018.

3. Chittester B. Medical device clinical trials-how do they compare with drug trials? 2014. https://www.mastercontrol.com/gxp-lifel ine/medical-device-clinical-trials-how-do-they-compare-withdrug-trials. Accessed May 23, 2018.

4. The Clinical Trial Company Group. The challenges in medical device clinical trials. https://www.tctcgroup.com/singl e-post/2018/01/23/The-challenges-in-medical-device-clinical-trial s. Accessed 25 May 2018.

5. Fearon WF, Bornschein B, Tonino PA, Gothe RM, Bruyne BD, Pijls $\mathrm{NH}$, et al. Economic evaluation of fractional flow reserveguided percutaneous coronary intervention in patients with multivessel disease. Circulation. 2010;122(24):2545-50.

6. Redekop WKMJ, Verboom P, Lovas K, Kalo Z, Redekop WK, McDonnell J, Verboom P, Lovas K, Kalo Z. The cost effectiveness of Apligraf treatment of diabetic foot ulcers. Pharmacoeconomics. 2003;21(16):1171-83.

7. Shen LJ, Chou H, Huang CF, Chou GM, Chan WK, Wu FL. Economic benefits of sponsored clinical trials on pharmaceutical expenditures at a medical center in Taiwan. Contemp Clin Trials. 2011;32(4):485-91. https://doi.org/10.1016/j.cct.2011.04.003.

8. Bredin C, Eliasziw M, Syme R. Drug cost avoidance resulting from cancer clinical trials. Contemp Clin Trials. 2010;31(6):5249. https://doi.org/10.1016/j.cct.2010.09.004.5.

9. Medical Devices Harm Patients Worldwide As Governments Fail On Safety. November 25, 2018. https://www.icij.org/investigat ions/implant-files/medical-devices-harm-patients-worldwide-asgovernments-fail-on-safety/. Accessed 28 Jan 2019.

10. U.S. National Library of Medicine Clinical Trial Database. https ://clinicaltrials.gov/. Accessed 25 May 2018.

11. Canada-Healthcare and Medical equipment. https://www.expor t.gov/article? $\mathrm{id}=$ Canada-Healthcare-andMedical-Equipment. Accessed 28 Jan 2018.

12. Medical Product Outsourcing. Top 30 Global Medical Device Companies. https://www.mpo-mag.com/issues/2017-07-01/ view_features/top-30-global-medical-device-companies-46193 4. Accessed May 28, 2018.

13. Loo J. 2013 S\&P Capital IQ Industry Surveys. Healthcare: Products\&Supplies. https://gskkr.files.wordpress.com/2015/01/ healthcare-products-and-services.pdf. Accessed 23 May 2018.

14. Alberta Health Services. https://www.albertahealthservices.ca/. Accessed 16 June 2018. 
15. Northern Alberta Clinical Trials and Research Centre. NACTRC. https://nactrc.ca/. Accessed 21 Jan 2018.

16. Tran DT, Akpinar I, Fedorak R, Jonsson E, Mackey J, Richer L, et al. The economic contribution of industry-sponsored pharmaceutical clinical trials. J Pharm Pharm Sci. 2017;20(1):407-14.

17. Akpinar I, Jacobs P, Tran TD. Forecasting pharmaceutical prices for economic evaluations when there is no market: a review. Pharmacoecon Open. 2017;1(1):65-8.

18. Purchasing Power Parities. Organisation for Economic Cooperation and Development- OECD data. https://data.oecd.org/ conversion/purchasing-power-parities-ppp.htm\#indicator-chart. Accessed 1 June 2018.

19. Grimm SE, Stevens JW. When future change matters: modeling future price and diffusion in health technology assessments of medical devices. Value Health. 2016;19(6):720-6.

20. Alberta Inflation Calculator. http://inflationcalculator.ca/alberta/. Accessed 21 June 2018

21. Mealing S, Woods B, Hawkins N, Cowie MR, Plummer CJ, Abraham WT, et al. Cost-effectiveness of implantable cardiac devices in patients with systolic heart failure. Heart. 2016;102(21):1742-9.

22. Gatlin SW, Kim DW, Mahle WT. Cost analysis of percutaneous pulmonary valve replacement. Am J Cardiol. 2011;108(4):572-4.

23. Baxter Price List \& Product Catalog 2011. www.baxter.com.sg/ downloads/patients_and_caregivers/therapies/renal/bplpc.pdf. Accessed 23 May 2018.
24. Gladwell DHT, Cook M, Akehurst R. Cost effectiveness of renal denervation therapy for the treatment of resistant hypertension in the UK. Appl Health Econ Health Policy. 2014;12(6):611-22.

25. Geisler BP, Egan BM, Cohen JT, Garner AM, Akehurst RL, Esler $\mathrm{MD}$, et al. Cost-effectiveness and clinical effectiveness of catheter-based renal denervation for resistant hypertension. J Am Coll Cardiol. 2012;60(14):1271-7.

26. The Canadian Agency for Drugs and Technologies in Health. CADTH 2014 Common Drug Review Somatropin (Genotropin) for subcutaneous injection. https://www.cadth.ca/media/cdr/.../ SR0333_GenotropinGHD-P_CL_Report_e.pdf. Accessed 23 May 2018.

27. Inc. SM. Spectral Medical Inc.Targeted therapy for septic shock. 2018. http://www.spectraldx.com/assets/spectral_investor_updat e_0518.pdf. Accessed 21 May 2018.

28. BTG Interventional Medicine. https://www.btg-im.com/en-CA. Accessed 24 Apr 2018.

29. Wenzl M, Mossialos E. Prices for cardiac implant devices may be up to six times higher in the US than in some European countries. Health Aff (Millwood). 2018;37(10):1570-7.

30. The sponsor: responsibilities in medical device clinical trialsFDA. https://www.fda.gov/downloads/training/cdrhlearn/ucm17 6457.pdf. Accessed 29 June 2018. 